Submitted to ApJ

Preprint typeset using LTEX style emulateapj v. 5/2/11

\title{
PREVENTING STAR FORMATION IN EARLY-TYPE GALAXIES WITH LATE-TIME STELLAR HEATING
}

\author{
Charlie Conroy $^{1,2}$, Pieter G. VAn DokKum ${ }^{3}$, Andrey Kravtsov ${ }^{4,5}$ \\ Submitted to ApJ
}

\begin{abstract}
We revisit previous suggestions that the heating provided by the winds of dying low-mass stars plays an important role in preventing star formation in quiescent galaxies. At the end of their asymptotic giant branch phase, intermediate and low-mass stars eject their envelopes rapidly in a super-wind phase, usually giving rise to planetary nebulae. In spheroidal galaxies with high stellar velocity dispersions, the interaction of these ejected envelopes with the ambient diffuse gas can lead to significant, isotropic and steady-state heating that scales as $\dot{M}_{*} \sigma_{*}^{2}$. We show that cooling of the central regions of the hot diffuse halo gas can be delayed for a Hubble time for halos more massive than $\sim 10^{12.5} M_{\odot}$ at $0<z<2$, although stellar heating alone is unlikely to forestall cooling in the most massive clusters at $z=0$. This mechanism provides a natural explanation for the strong trend of galaxy quiescence with stellar surface density and velocity dispersion. In addition, since the ejected material will thermalize to $k T \sim \sigma_{*}^{2}$, this mechanism provides an explanation for the observed similarity between the central temperature of the hot diffuse gas and $\sigma_{*}^{2}$, a result which is not trivial in light of the short inferred cooling times of the hot gas. The main uncertainty in this analysis is the ultimate fate of the stellar ejecta. Preventing accumulation of the ejecta in the central regions may require energy input from another source, such as Type Ia supernovae. Detailed simulations of the interaction of the stellar wind with the ambient gas are required to better quantify the net effect of AGB heating.
\end{abstract}

Subject headings: galaxies: stellar content — galaxies: elliptical and lenticular, $\mathrm{cD}$ — galaxies: evolution

\section{INTRODUCTION}

Galaxies with little-to-no star formation are common today and have been discovered at remarkably early epochs $(z=2-4$; e.g., Daddi et al. 2005; Kriek et al. 2006; Onodera et al. 2012; Whitaker et al.2013). The existence of these quiescent galaxies poses two major questions to galaxy formation models: what causes the cessation of star formation (quenching) and what maintains the low observed star formation rates over cosmological timescales. The second issue is challenging because massive quiescent galaxies are embedded in dark matter halos containing hot diffuse gas that, in the absence of a heat source to offset radiative cooling, will eventually form new stars (e.g., Fabian et al. 1984, Croton et al. 2006). Hydrodynamic simulations with standard recipes for gas cooling and energy feedback from Type II supernovae are generally unable to produce realistic quiescent galaxies (e.g., Martizzi et al. 2014).

A variety of heating sources have been proposed in order to offset the radiative losses of the diffuse gas, although by far the most popular is some form of energetic feedback from an active galactic nucleus (AGN). Evidence that supermassive black holes do something to the diffuse gas in low redshift clusters is ubiquitous, including the existence of giant radio cavities, sound waves, non-thermal pressure support, etc. (see McNamara \& Nulsen 2007, for a recent review). However, it has been difficult to convincingly demonstrate that this activity is directly responsible for heating the diffuse gas.

\footnotetext{
${ }^{1}$ Department of Astronomy \& Astrophysics, University of California, Santa Cruz, CA, USA

2 Department of Astronomy, Harvard University, Cambridge, MA, USA

${ }^{3}$ Department of Astrophysical Sciences, Yale University, New Haven, CT, USA

${ }^{4}$ Department of Astronomy \& Astrophysics, 5640 South Ellis Ave., The University of Chicago, Chicago, IL 60637

${ }^{5}$ Kavli Institute for Cosmological Physics and Enrico Fermi Institute, 5640 South Ellis Ave., The University of Chicago, Chicago, IL 60637
}

Recently it has become clear that galaxy quenching is more strongly correlated with their central stellar densities and velocity dispersions, than their overall stellar mass (e.g., Kauffmann et al. 2003, Franx et al. 2008, Bell et al. 2012, Cheung et al. 2012; Fang et al. 2013). This is particularly striking at $z \sim 2$, where $\sim 50 \%$ of galaxies dwith $M_{*}>10^{11} M_{\odot}$ are quiescent and $\sim 50 \%$ are star forming (Brammer et al.2011) and the stellar density is an excellent predictor of star formation rate (Franx et al. 2008). This suggests that the stars may play an important role in either quenching galaxies or maintaining the low observed star formation rates. Morphological quenching is one possible mechanism, in which the high velocity dispersion stabilizes a gaseous disk against fragmentation (e.g., Martig et al. 2009). However, in this scenario the cooling of the hot gas would likely result in the accumulation of giant gas disks that would eventually become unstable.

In this paper we consider the possibility that the old stars themselves are responsible for heating the gas, thus potentially obviating the need for a separate mechanism (such as AGN feedback) to explain the strong correlation between high stellar densities and the absence of star formation. We will focus on mass loss by AGB stars, both in isolation and in tandem with the effects of Type Ia supernova explosions. In this scenario the heating rate scales directly with both the local stellar density and the stellar velocity dispersion squared (the latter arises because of the thermalization of the stellar winds). These heating sources are an inevitable consequence of stellar evolution; the main questions are whether there is enough energy to offset radiative cooling and the extent to which wind material accumulates in the central regions of galaxies.

It has long been recognized that dying low-mass stars and Type Ia SNe may contribute to the heating of diffuse gas in quiescent galaxies (e.g., Mathews \& Baker 1971; Lake \& Schommer 1984; Mathews 1990, Ciotti et al. 1991; Matsushita 2001). Much of the early work focused on questions relating to the origin of hot gas and cooling flows in massive 


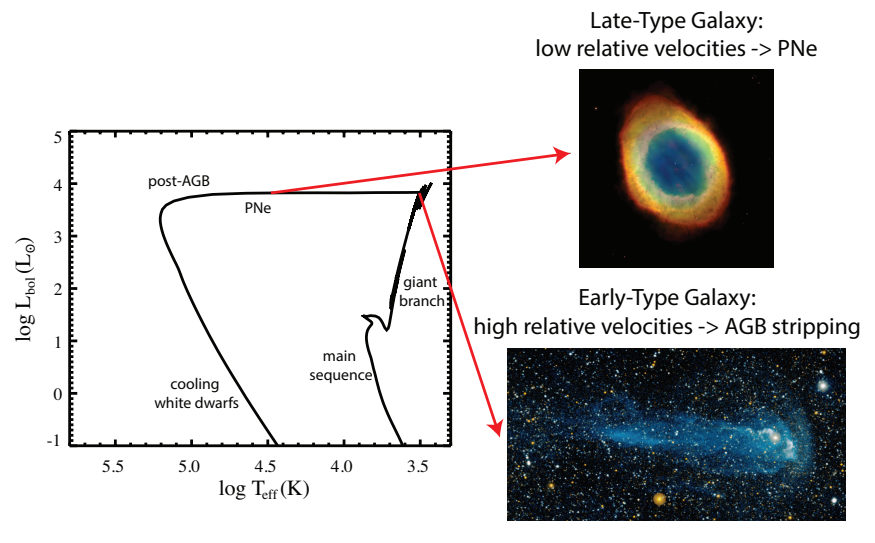

Figure 1. Schematic illustration of the thermalization of winds from low mass stars. When AGB stars move with low velocity relative to the surrounding gas, ejected material remains near to the central star, creating planetary nebulae (such as the Ring Nebula shown in the top right panel from the Hubble Heritage Team). However, when AGB stars move with high velocities relative to the ambient gas, the ejected matter is swept away by ram pressure such as seen in the UV image of Mira in the bottom right panel; image credit: NASA/JPL-Caltech/C. Martin/M. Seibert. Though Mira is in the Milky Way, its velocity of $130 \mathrm{~km} \mathrm{~s}^{-1}$ relative to the ambient gas (Martin et al. 2007) is a configuration expected to be common in the central regions of early-type galaxies. In this case the energy required to decelerate the ejected material is transfered to the background gas, which can result in a net heating of the gas with $\sigma_{*}^{2} \gtrsim T$.

systems. Here we re-examine these heating mechanisms in the context of current galaxy formation models to determine whether they are sufficiently powerful to explain the low observed star formation rates in quiescent galaxies, and especially in the population of compact quiescent galaxies at high redshift.

\section{BASIC ARGUMENTS \\ 2.1. Stellar Wind Heating}

We begin by considering the heating due to late-time stellar mass loss from evolved low mass stars. Consider a region with stellar density, $\rho_{*}$. A stellar population of initial mass $M_{*}$ and age $t$ loses mass at a rate that can be approximated as $\dot{M}_{*} \approx 8\left(M_{*} / M_{\odot}\right)(t / \mathrm{yr})^{-1.25} M_{\odot} \mathrm{yr}^{-1}$ for ages $>10^{7} \mathrm{yrs}$, and a Chabrier (2003) IMF (estimated from the models of Conroy et al. 2009). Much, perhaps most, of the mass lost by old stellar populations is due to a single phase of stellar evolution: near the end of the AGB phase, stars undergo a catastrophic mass-loss event in which they shed their remaining envelope (Iben \& Renzini 1983) ${ }^{6}$ The ejection of the envelope often leads to the formation of a planetary nebula. The cold gaseous envelope shed in this stage shares the velocity of the parent star relative to the ambient gas and will thus interact with the gas (e.g., Mathews 1990). The work done by the background gas to decelerate the circumstellar shell may result in a net heating of the background gas. See Figure 1 for a schematic illustration.

In addition to heating the surrounding ambient gas, some energy must also go into heating the stellar ejecta in order for late-time stellar heating to be effective. In fact, when $T>\sigma_{*}^{2}$, the interaction of the AGB ejecta with the ambient gas will result in net cooling, not heating. However, if cooling brings

6 Whether or not the majority of the mass-loss occurs very rapidly or slowly (as might be the case for RGB mass-loss) is immaterial for our purposes because in both cases the background gas must do work to decelerate the ejected material to zero relative velocity. the temperature of the ambient gas substantially below $\sigma_{*}^{2}$, the thermalization of AGB ejecta will heat the gas back to $\sigma_{*}^{2} \sim$ $T$. In the calculations that follow we assume the maximum heating rate for the case when $T \ll \sigma_{*}^{2}$. In $\$ 4$ we mention the possibility that AGB heating may ultimately be responsible for the observed similarity between $T$ and $\sigma_{*}^{2}$ for groups and clusters.

If the entire kinetic energy of the lost gas were transferred to internal energy of the ambient gas, the heating rate per unit volume would be

$$
\dot{e}_{\text {heat }}=\frac{1}{2} \frac{\dot{M}_{*} \sigma_{*}^{2}}{V}=\frac{1}{2} \frac{8 M_{*} \sigma_{*}^{2}}{V t^{1.25}}=\frac{4 \rho_{*} \sigma_{*}^{2}}{t^{1.25}} .
$$

Here $V$ is a volume element and $\sigma_{*}$ is the three-dimensional velocity dispersion of stars.

The cooling rate per unit volume of the ambient gas of number density $n_{g}$ is $\dot{e}_{\text {cool }}=n_{g}^{2} \Lambda(T, Z)$, where $\Lambda(T, Z)$ is the cooling function depending on temperature and metallicity. Herein we adopt the cooling function of Sutherland \& Dopita (1993).

The ratio of heating to cooling rate is then:

$$
\frac{\dot{e_{\text {heat }}}}{\dot{e}_{\text {cool }}}=\frac{4 \rho_{*} \sigma_{*}^{2}}{t^{1.25} n^{2} \Lambda(T, Z)} \approx 10 \rho_{* 1} \sigma_{*, 300}^{2} n_{g,-2}^{-2} \Lambda_{-23}^{-1} t_{9.7}^{-1.25},
$$

where $\rho_{*, 1}$ is in units of $M_{\odot} \mathrm{pc}^{-3}, \sigma_{*, 300}$ is the velocity dispersion in units of $300 \mathrm{~km} / \mathrm{s}, n_{g,-2}$ is the ambient gas number density in units of $10^{-2} \mathrm{~cm}^{-3}, \Lambda_{-23}$ is the cooling function in units of $10^{-23} \mathrm{erg} \mathrm{s}^{-1} \mathrm{~cm}^{3}$ and $t_{9.7}$ is the stellar population age in units of $5 \times 10^{9} \mathrm{yrs}$, which is typical for an average age of stellar populations of both the inner regions of $z \sim 0$ disk galaxies and ellipticals. For the temperature range relevant for ambient halo gas, $T \sim 10^{5}-10^{7} \mathrm{~K}$ of metallicity, $Z \sim 0.01-1.0 Z_{\odot}, \Lambda$ ranges from $\sim 10^{-23}$ to $\sim 10^{-22}$ (e.g., Sutherland \& Dopita 1993).

Finally, the AGB heating expressed as a total power available is

$$
\dot{E}_{\mathrm{AGB}} \approx 5 \times 10^{40} M_{*, 11} \sigma_{*, 300}^{2} t_{9.7}^{-1.25} \mathrm{erg} \mathrm{s}^{-1},
$$

where $M_{*, 11}$ is the stellar mass in units of $10^{11} M_{\odot}$.

We infer that the heating rate from stellar mass loss could, in this optimistic scenario, exceed the cooling rate by a factor of $\sim 10$ for massive compact galaxies.

In these calculations we have ignored the fact that stellar ejecta will accumulate in the central regions. If left unchecked, the growing central gas density will increase the cooling rate of the ambient gas, eventually to the point where cooling may overwhelm AGB heating. This issue is addressed in the next section.

\subsection{Type Ia SNe Heating}

We can compare the energy available in wind thermalization to the total energy available from another significant latetime stellar heating source: Type Ia SNe explosions. The delay time distribution of type Ia $\mathrm{SNe}$ has been estimated by Maoz et al. (2012) to be $\approx 3 \times 10^{-2} t^{-1.1} \mathrm{SNe}$ per year per $M_{*, 11}$ where $t$ is in Gyr. If we adopt an energy deposition per event of $10^{51} \mathrm{erg}$, then the power available from Ia SNe is:

$$
\dot{E}_{\mathrm{Ia}} \approx 14 \times 10^{40} M_{*, 11} t_{9.7}^{-1.1} \mathrm{erg} \mathrm{s}^{-1} .
$$

Notice that the time and mass dependence of the two forms of heating are very similar and for massive galaxies they are comparable. Both sources will generally work in tandem to 
heat the gas, with their relative importance a function of velocity dispersion: the ratio of these energy sources scales as $\sigma_{*}^{2}$, such that in galaxies with velocity dispersions less than $\sim 300 \mathrm{~km} \mathrm{~s}^{-1}$ the heating due to Ia SNe is much larger than the heating due to wind thermalization. We expect AGB heating to dominate in massive and compact galaxies.

Furthermore, notice that the binding energy of the accumulated wind material evolves as:

$$
\dot{E}_{g}=\frac{G M_{*} \dot{M}_{*}}{R_{*}} \approx 9 \times 10^{40} M_{*, 11} t_{9.7}^{-1.25} R_{*, 1}^{-1} \mathrm{erg} \mathrm{s}^{-1},
$$

where $R_{*, 1}$ is the galaxy half-mass size in units of $1 \mathrm{kpc}$. The thermal energy provided by type Ia SNe explosions should therefore be sufficient to unbind the accumulated material.

In the rest of this article we will refer to 'AGB heating' and 'late-time stellar heating' interchangeably, but a more precise phrase would be 'SNe Ia-assisted AGB heating' to emphasize that the clearing of accumulated material by Ia $\mathrm{SNe}$ is an important aspect of this scenario.

\section{MASS AND REDSHIFT DEPENDENCE OF LATE-TIME STELLAR HEATING}

\subsection{Expectations}

We now proceed to compute the ratio between AGB heating and radiative cooling (i.e., Equation 2 as a function of stellar mass, $M_{*}$, dark matter halo mas: $7 M_{\text {halo }}$, and redshift. Based on the arguments in the previous section, we assume that the accumulated AGB ejecta is cleared from the central regions of the galaxy by type Ia SNe. Here we are interested in global quantities, and so we replace $\rho_{*}$ with $M_{*}$ and $n_{g}$ with $N_{g}$ (total number of gas particles). The former is a relatively well-defined quantity but the latter requires a choice of radius. Here we adopt a radius of $r_{\text {cool }}=r_{* \text {,half }}$, where $r_{* \text {,half }}$ is the half-mass radius of the stars. We regard AGB heating to be effective if it can offset radiative losses within the cooling radius, $r_{\text {cool }}$. The cooling radius is the most important adopted parameter in what follows because the total cooling rate scales as $r_{\text {cool }}^{3}$. We consider alternative definitions of $r_{\text {cool }}$ below.

For the properties of the galaxies and the stars within them, we make the following assumptions. We adopt the $M_{*}-\sigma_{*}$ relation at $z=0$ for early-type galaxies from Dutton et al. (2011), which is a complex function but basically scales as $M_{*} \propto \sigma_{*}^{3.5}$. We assume that at fixed $\sigma_{*}$, the stellar mass of galaxies decrease by a factor of two from $z=0$ to $z=2$ (e.g., Belli et al. 2014). Note that observations report a one dimensional velocity dispersion; we multiply the reported values by $\sqrt{3}$ to approximate the three dimensional dispersion. We also adopt the $z=0$ mass-size relation for early-type galaxies from Dutton et al. (2011) and scale it by $(1+z)^{-1}$ for higher redshifts (van der Wel et al.2014). We also must specify a typical age of the stars in order to set the mass-loss rate. Here we use the $M_{*}$-stellar age relation at $z=0$ from Choi et al. (2014), and we scale the ages by the Hubble time as a function of redshift. We adopt a minimum age of $2 \mathrm{Gyr}$ as we do not expect quiescent galaxies to have ages considerably younger than this, by definition.

For the diffuse halo gas we adopt the following model. We assume the diffuse gas is isothermal and at the halo virial temperature, $T_{\mathrm{vir}}$, which for groups and clusters at $z=0$ is accu-

\footnotetext{
${ }^{7}$ Halo masses are defined according to the Bryan \& Norman (1998) virial definition. Where necessary a normalized Hubble constant of $h=0.7$ is adopted.
}
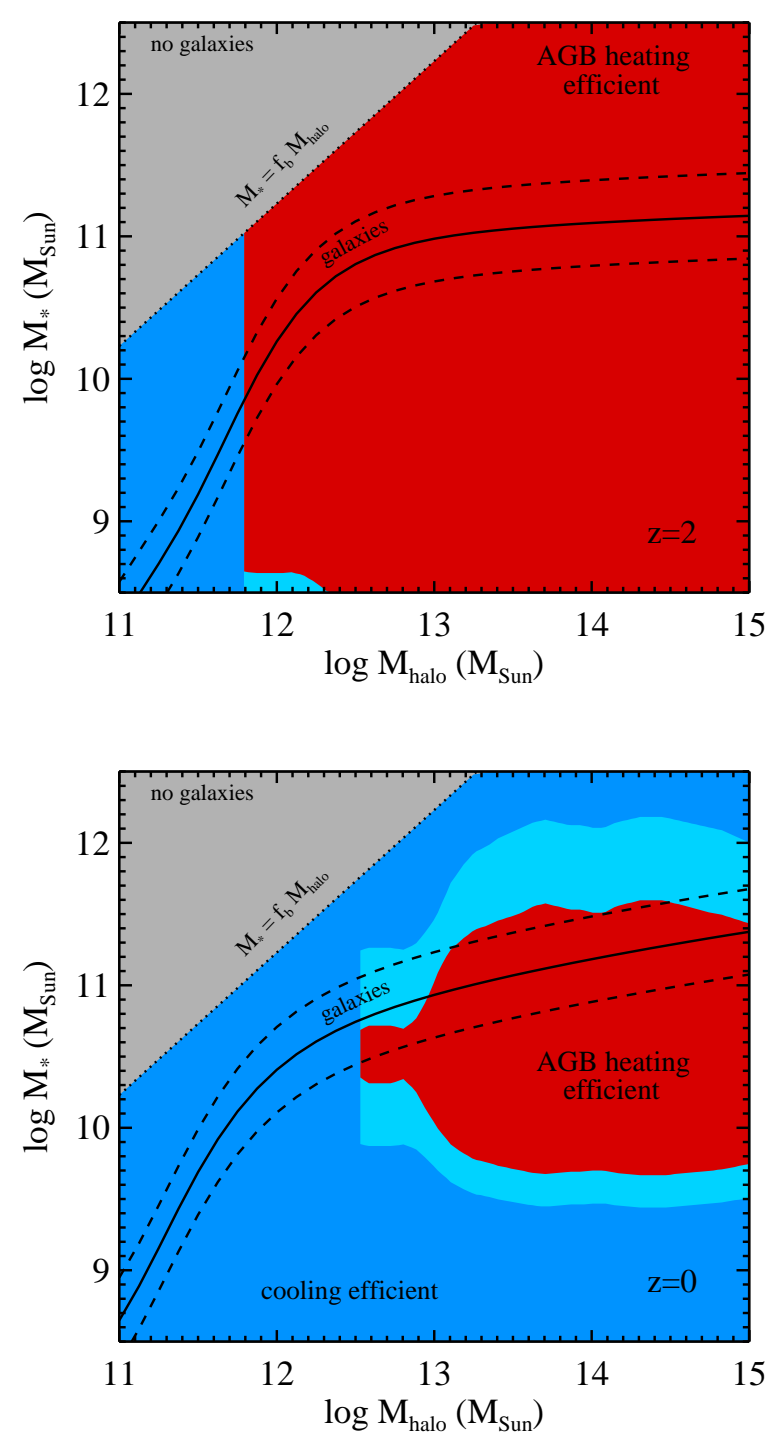

Figure 2. Potential efficiency of AGB heating. This diagram compares radiative cooling of diffuse halo gas to AGB heating as a function of galaxy stellar mass, $M_{*}$, and halo mass, $M_{\text {halo }}$, at $z=2$ (top panel) and $z=0$ (bottom panel). Red and blue regions denote where AGB heating and radiative cooling dominate, respectively. The light blue region marks where heating and cooling balance to within a factor of two. Galaxies occupy the region within the dashed lines (Behroozi et al. 2013). The sharp vertical boundary between heating and cooling is a consequence of our assumption that halos with $T_{\text {vir }}<10^{6} \mathrm{~K}$ are always able to cool efficiently.

rate to within a factor of $\sim 1.5-2.5$ (Vikhlinin et al. 2006 , Sun et al. 2009). For halos with $T_{\text {vir }}<10^{6} \mathrm{~K}$ we assume that there is no stable hot halo and that available gas is able to settle into a cold disk. The diffuse gas has a metallicity of $\frac{2}{3} Z_{\odot}$, which is appropriate for the central regions of clusters (Rasmussen \& Ponman 2007; Johnson et al. 2011; Sasaki et al. 2014). The choice of the diffuse gas density is a second (following $r_{\text {cool }}$ ) key model parameter. Here we assume that the ambient gas density is constant within $r_{\text {cool }}$ and with redshift and equal to $10^{-2} \mathrm{~cm}^{-3}$. Empirically this is a good approximation for groups and clusters at $z=0$ (Vikhlinin et al.2006, Sun et al.2009). For lower mass systems the gas density is not well-constrained but a variety of arguments, including results from simulations, favor values of $n \sim 10^{-2} \mathrm{~cm}^{-3}$ or lower (e.g., 

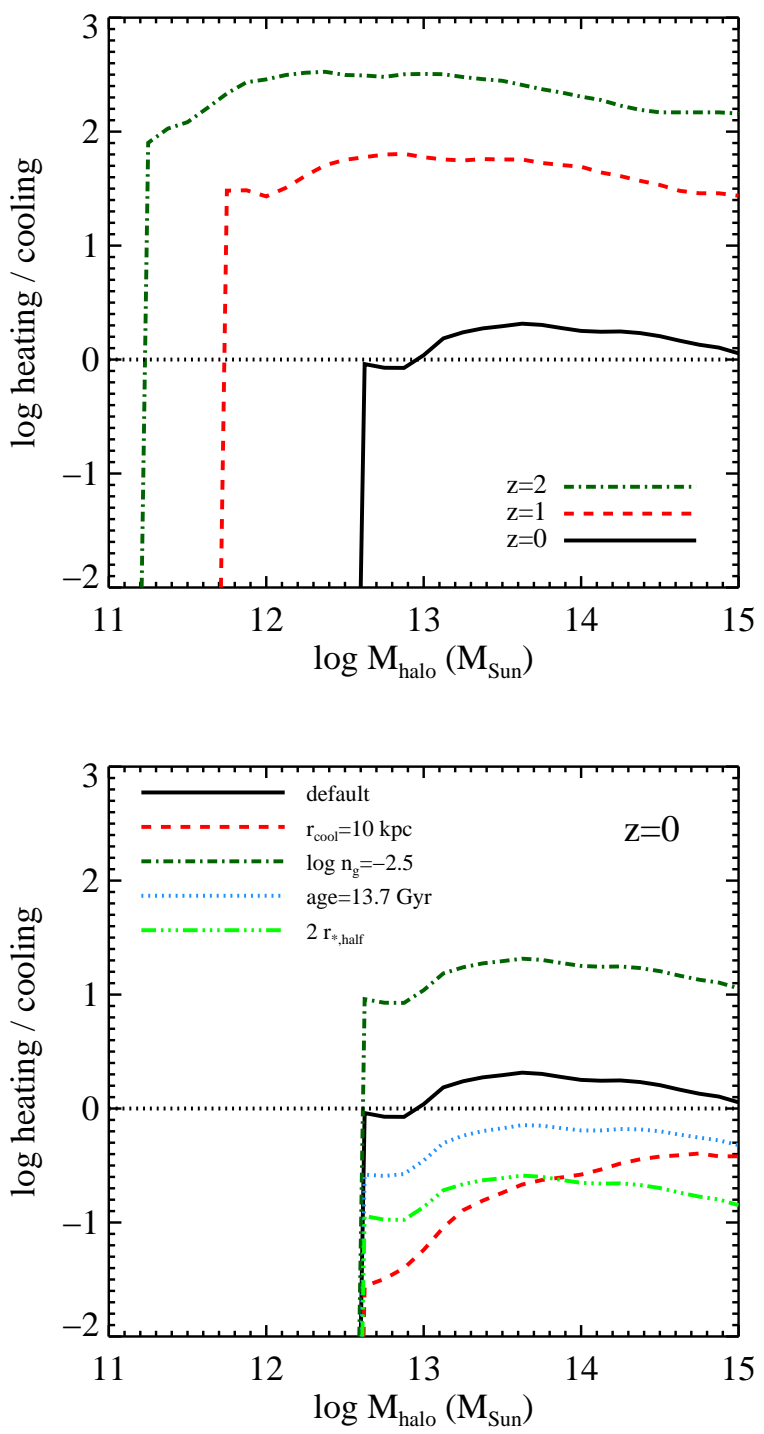

Figure 3. Balance between AGB heating and radiative cooling as a function of halo mass. At each halo mass the heating and cooling rates are computed for the galaxy expected to occupy the halo. Top panel: Results for our default model assumptions at $z=0,1,2$. Bottom panel: Results after varying several model assumptions at $z=0$. The variations include fixing the cooling radius to $10 \mathrm{kpc}$ and setting $r_{\text {cool }}=2 r_{* \text {,half }}$, decreasing the gas density to $10^{-2.5} \mathrm{~cm}^{-3}$ and fixing the stellar ages to $13.7 \mathrm{Gyr}$ for all galaxies.

Guedes et al. 2011; Feldmann et al. 2013; Agertz et al. 2013). A constant gas density within $r_{\text {cool }}$ is a reasonable assumption here since $r_{\text {cool }}$ is proportional to $r_{* \text { half }}$, and the latter is proportional to the halo virial radius (Kravtsov 2013). The cooling radius in our model is therefore tracking a fixed fraction of the halo virial radius, and assuming a self-similar gas density profile, one might expect the mean density within $r_{\text {cool }}$ to be constant with mass and redshift.

With the assumptions above we are now ready to compute the balance between AGB heating and radiative cooling as a function of $M_{*}$ and $M_{\text {halo }}$ at various redshifts. We show this balance in Figure 2 Red regions in this diagram indicate where AGB heating is effective at offsetting radiative losses. We also show the expected locations of galaxies based on the abundance matching technique (Behroozi et al. 2013).

The main result of this paper is that AGB heating can be an efficient mechanism to offset radiative losses from diffuse gas over much of the $M_{*}-M_{\text {halo }}$ plane, and certainly in the regime where quiescent galaxies reside (roughly above the bend in the galaxy $M_{*}-M_{\text {halo }}$ relation). This mechanism is more efficient at high redshift for several reasons: 1) galaxies are more compact at higher redshift, and so the adopted cooling radius is smaller; 2) at fixed stellar mass galaxies have higher velocity dispersions at higher redshift; and 3) the stellar mass-loss rates are higher at high redshift. We emphasize here that we are considering the ability of AGB heating to offset cooling of hot diffuse gas. At low halo masses the majority of the gas is expected to be cool and dense, and so we do not expect AGB heating to compete with radiative cooling (see also $\$ 3.2$ below).

AGB heating is unlikely to be effective at shutting down star formation in the cold interstellar medium (ISM). The densities of the cold ISM are several orders of magnitude higher than the limit where post-AGB heating is effective at offsetting radiative cooling (see Figure 4 below). Furthermore, in dynamically cold systems the relative velocities between the gas and the stars are in general low, which means that the amount of energy transfered from the circumstellar envelope to the gas will be low.

Figure 3 shows the balance between AGB heating and radiative cooling as a function of $M_{\text {halo }}$. At each halo mass the heating and cooling rates are computed for the galaxy expected to occupy the halo. The top panel shows our default model assumptions at $z=0,1,2$ while the bottom panel shows results after varying several model assumptions. At $z=1,2$ the heating rates are very high both because the stellar ages are young and because the stellar densities and velocity dispersions are high at a fixed $M_{*}$. As expected, $r_{\text {cool }}$ and $n_{g}$ have a significant effect on our results which is not surprising because the total cooling rate scales as $r_{\text {cool }}^{3} n_{g}^{2}$.

\subsection{Comparison to Observations}

Figure 4 shows the region where AGB heating is effective at a local level, in the gas-stellar density plane. In this diagram we show the expected locus of $z \sim 2$ quiescent galaxies, the observed locations of central galaxies within $z=0$ groups and clusters, the Milky Way bulge, stellar and gas density profiles from the THINGS survey, and expected locations of typical star-forming galaxy disks at $0<z<1$. We estimate the latter by adopting mass-size relations for disks from van der Wel et al. (2014), the mass-SFR relation from Whitaker et al. (2012), and a fixed gas depletion timescale of 2 Gyr (defined as $\left.\tau_{\text {dep }} \equiv \Sigma_{\text {gas }} / \Sigma_{\text {SFR }}\right)$. We also assume that the gas disk scale length is twice that of the stars and the scale heights of the gas and disk are $10 \%$ of the corresponding scale lengths.

The number density of the hot halo gas in the inner few kpc of Milky Way-like galaxies is expected to be close to $n \sim 10^{-2} \mathrm{~cm}^{-3}$ (e.g., Guedes et al. 2011, Feldmann et al. 2013). Figure 4 shows that for these fiducial values AGB heating can offset cooling for stellar densities $\rho_{*} \gtrsim 1 M_{\odot} \mathrm{pc}^{-3}$ assuming $\sigma_{*}=100 \mathrm{~km} \mathrm{~s}^{-1}$. For disk galaxies of a typical scale height $h_{*} \approx 200$ pc (Bovy \& Rix 2013), such densities would correspond to a surface density of $\Sigma_{*} \sim 4 \rho_{*} h_{*} \sim 800 M_{\odot} \mathrm{pc}^{-2}$, which is close to the surface densities that separate blue star forming galaxies at $z \approx 0$ from the galaxies in the green valley and red sequence (Fang et al.2013).

Another way to explore the balance between stellar ejecta heating and radiative cooling would be to compare $\dot{M}_{*} \sigma_{*}^{2}$ directly to the X-ray luminosity within a radius where the cool- 


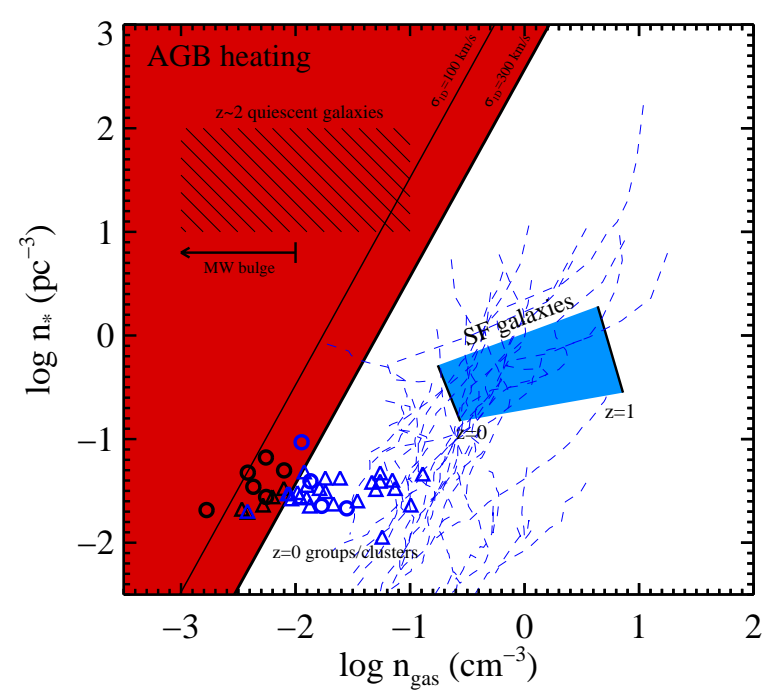

Figure 4. Local efficiency of AGB heating compared to radiative cooling as a function of gas and stellar density (red denotes regions where heating is dominant). Also shown is the expected location of star forming (SF) galaxies at $0<z<1$ and the approximate locus of quiescent galaxies at $z=2$. For the latter, the range of gas densities shown is the expected central gas densities for gas in hydrostatic equilibrium with dark matter halos where the gas is at the halo virial temperature. Dashed lines are radial gas and stellar profiles of disk galaxies from the THINGS survey (Leroy et al.2008). Also shown is the location of the central $\sim 10 \mathrm{kpc}$ of local galaxy groups (Sun et al. 2009 open circles) where $\mathrm{H} \alpha$ imaging (McDonald et al. 2011) shows evidence for either no emission (black symbols) or extended emission (blue symbols), and galaxy clusters from Haarsma et al. (2010 triangles) color-coded by the central cooling time ( $>10^{9.5} \mathrm{yr}$ in black and younger in blue).

ing times are short. Matsushita (2001) and Nagino \& Matsushita (2009) have made precisely this comparison for $\sim 50$ early-type galaxies in field and cluster environments. These authors find that in nearly all cases (excluding $\mathrm{cD}$ galaxies in massive clusters) the kinetic heating rate by stellar mass loss is comparable to or greater than the observed $\mathrm{X}$-ray luminosity. Furthermore, Nagino \& Matsushita (2009) find the intriguing result that the temperature gradient in the ambient gas is correlated with the ratio of $L_{B} \sigma^{2}$ to X-ray luminosity. This suggests that stellar ejecta and SNe Ia heating, both of which should correlate with $L_{B} \sigma^{2}$ (though in different ways), play an important role in shaping the properties of the ambient gas.

Massive quiescent galaxies at $z \sim 2$ are remarkably compact, with typical sizes of $\sim 1 \mathrm{kpc}$ and one dimensional velocity dispersions of $\gtrsim 300 \mathrm{~km} \mathrm{~s}^{-1}$ (e.g., van Dokkum et al. 2009, Belli et al. 2014). The implied stellar densities exceed $10 M_{\odot} \mathrm{pc}^{-3}$. For these galaxies, the heating rate of the diffuse gas due to AGB wind deceleration may exceed radiative losses by factors of $10-100$. In fact, at the extreme end of stellar densities and velocity dispersions observed in high redshift quiescent galaxies, AGB heating may disrupt even the cold ISM and might therefore play a role in shutting down star formation in these extraordinary systems.

The mechanism proposed here may explain the results reported in Salim et al. (2012), who found that low-level star formation on the optically-defined red sequence was confined mostly to S0 galaxies - true ellipticals rarely showed UV signatures of star formation. AGB heating in SOs will not be isotropic and the mass-loss will be largely confined to a plane, which may actually fuel the observed low-level star forma- tion.

\section{DISCUSSION \& CONCLUSIONS}

The mechanism described here is a necessary consequence of stellar evolution, yields a direct, steady-state, and approximately isotropic heating of the gas, and provides a natural explanation for the observed tight correlations between the stellar density of galaxies and their star formation rate (e.g., Kauffmann et al. 2003, Franx et al. 2008; Bell et al. 2012, Cheung et al. 2012, Fang et al. 2013). In all these respects it is a viable alternative to AGN feedback, which is the leading proposed mechanism for maintaining the low star formation rates in quiescent galaxies (Croton et al. 2006). We emphasize, however, that AGB heating likely cannot explain why star formation ceases in the first place (except perhaps in the very densest and highest velocity dispersion galaxies): it may be that the star forming progenitors of quiescent galaxies simply consume all of the available cold gas (Feldmann \& Mayer 2015), or that AGN feedback is a source of initial heating and/or removal of cold gas. Late-time stellar heating may then provide the long-term "maintenance" to keep dead galaxies dead.

There are other potential heating processes associated with old stellar populations. The most widely discussed such process is heating by type Ia supernovae (e.g., Mathews 1990, Ciotti et al. 1991). As shown in \$2.1. for massive galaxies the heating rate from $\mathrm{SNe}$ Ia is comparable to the AGB heating rate. The scaling with stellar population age and mass is similar for both heating mechanisms. However, Ia heating is insensitive to the velocity dispersion whereas AGB heating scales as $\sigma_{*}^{2}$. SNe Ia heating will therefore dominate AGB heating at low velocity dispersions, while AGB heating will dominate in massive compact (high dispersion) galaxies. In this article we have employed Ia explosions to clear the accumulated AGB ejecta. In reality they of will also likely directly heat the ambient hot gas. It is beyond the scope of the present work to consider this second energy source in detail (see e.g., Ciotti et al. 1991; David et al. 2006, Negri et al.|2014, and references therein for discussion). Of course, any self-consistent model of galaxy evolution should include all heating sources, including type Ia SNe and AGB heating.

AGB heating may also provide a natural interpretation of the observed central temperatures of the hot gas in groups and clusters. X-ray observations of these systems has revealed that the temperature profiles drop in the central regions by a relatively modest amount, typically by a factor of $\sim 1.5-2.5$ relative to the maximum temperature found at larger radius (e.g., Vikhlinin et al. 2006; Sun et al. 2009). Moreover, the central temperature is close to $\sigma_{*}^{2}$ (Matsushita 2001; Nagino \& Matsushita 2009). These observations are surprising in light of the generally short cooling times $(\lesssim 1 \mathrm{Gyr})$ found in the central regions. The lack of vigorous star formation indicates that this gas is not cooling catastrophically, but it is not obvious why a generic heating mechanism, such as AGN or $\mathrm{SNe}$ feedback, would maintain a temperature so close to $\sigma_{*}^{2}$. Indeed, many forms of distributed heating have difficulty reproducing observed temperature profiles without fine tuning (e.g., Conroy \& Ostriker 2008). However, heating due to wind thermalization would naturally lead to near equality between the specific internal energy of the ambient gas $\left(\frac{3}{2} k T / \mu m_{p}\right)$ and the specific kinetic energy of the stars $\left(\frac{1}{2} \sigma_{*}^{2}\right)$. This can be tested by carefully comparing the kinematic profile of central galaxies to the temperature profiles of the hot gas as a function 
of halo mass.

The potentially significant role of heating due to stellar ejecta means that it is important to implement and test this mechanism in simulations of galaxy formation. Inclusion of secular stellar mass loss is also important because it may in certain contexts be an important source of star formation fuel during late stages of galaxy evolution (e.g., Leitner \& Kravtsov 2011; Voit \& Donahue 2011), and may also feed the central black hole (e.g., Ciotti \& Ostriker 2001). Standard implementations of stellar mass loss in galaxy formation simulations include only an instantaneous return of mass to the surrounding gas after a star formation event (e.g., Katz et al. 1996). This does not capture mass loss and heating due to old stellar populations discussed here. Although secular stellar mass loss is now increasingly included in galaxy formation simulations (e.g., Kravtsov \& Gnedin 2005, Stinson et al. 2006; Agertz et al. 2013; Vogelsberger et al. 2013), current simulations only account for the mass and momentum transfer of the ejecta, but not the heating due to dissipation of its kinetic energy. Although implementation of such heating in numerical simulations is, in principle, straightforward, care must be taken to ensure that energy injected due to the mass loss heating is not dissipated away excessively due to resolution effects.

The AGB heating scenario has a variety of observational implications. Stripping of the circumstellar shells should produce anti-correlations between the specific frequency of planetary nebulae and galaxy velocity dispersion, both within and among galaxies. This is supported by currently existing data (Coccato et al.2009) and is a strong prediction of the model, although the timescale over which the stripping takes place is uncertain and will determine whether or not the visibility of planetary nebulae is affected. As the mass loss rate decreases with stellar age, we expect the oldest galaxies to have the most difficulty maintaining low levels of star formation, and perhaps some galaxies will show evidence of recent rejuvenation. As discussed in the previous section, AGB heating in S0 galaxies will be less effective than in true ellipticals. In addition, we expect SFR activity to be correlated with a combination of stellar density and velocity dispersion and so outliers in the $\rho_{*}-\sigma_{*}$ plane should provide valuable insight. Nearby environments such as the bulge of M31 and Centaurus A may provide important constraints on the interaction between ejected stellar envelopes and the surrounding hot diffuse gas. Heating caused by the thermalization of stellar ejecta may be responsible for the close similarity between the specific kinetic energy of the stars and the specific internal energy of the ambient gas (e.g., Nagino \& Matsushita 2009).

We thank the referee for very helpful comments that improved the quality and clarity of the manuscript. We also acknowledge useful discussions with Luca Ciotti and Jerry Ostriker. CC is supported by Packard and Sloan Foundation Fellowships. CC and PvD acknowledge the Lorentz Center meeting "What Regulates Galaxy Evolution?" in April 2013 where this work was conceived.

\section{REFERENCES}

Agertz, O., Kravtsov, A. V., Leitner, S. N., \& Gnedin, N. Y. 2013, ApJ, 770, 25

Behroozi, P. S., Wechsler, R. H., \& Conroy, C. 2013, ApJ, 770, 57
Bell, E. F. et al. 2012, ApJ, 753, 167

Belli, S., Newman, A. B., \& Ellis, R. S. 2014, ApJ, 783, 117

Bovy, J. \& Rix, H.-W. 2013, ApJ, 779, 115

Brammer, G. B. et al. 2011, ApJ, 739, 24

Bryan, G. L. \& Norman, M. L. 1998, ApJ, 495, 80

Chabrier, G. 2003, PASP, 115, 763

Cheung, E. et al. 2012, ApJ, 760, 131

Choi, J. et al. 2014, ApJ, 792, 95

Ciotti, L., D'Ercole, A., Pellegrini, S., \& Renzini, A. 1991, ApJ, 376, 380

Ciotti, L. \& Ostriker, J. P. 2001, ApJ, 551, 131

Coccato, L. et al. 2009, MNRAS, 394, 1249

Conroy, C., Gunn, J. E., \& White, M. 2009, ApJ, 699, 486

Conroy, C. \& Ostriker, J. P. 2008, ApJ, 681, 151

Croton, D. J. et al. 2006, MNRAS, 365, 11

Daddi, E. et al. 2005, ApJ, 626, 680

David, L. P., Jones, C., Forman, W., Vargas, I. M., \& Nulsen, P. 2006, ApJ, 653, 207

Dutton, A. A., Conroy, C., van den Bosch, F. C., Simard, L., Mendel, J. T., Courteau, S., Dekel, A., More, S., \& Prada, F. 2011, MNRAS, 416, 322

Fabian, A. C., Nulsen, P. E. J., \& Canizares, C. R. 1984, Nature, 310, 733

Fang, J. J., Faber, S. M., Koo, D. C., \& Dekel, A. 2013, ApJ, 776, 63

Feldmann, R., Hooper, D., \& Gnedin, N. Y. 2013, ApJ, 763, 21

Feldmann, R. \& Mayer, L. 2015, MNRAS, 446, 1939

Franx, M., van Dokkum, P. G., Schreiber, N. M. F., Wuyts, S., Labbé, I., \& Toft, S. 2008, ApJ, 688, 770

Guedes, J., Callegari, S., Madau, P., \& Mayer, L. 2011, ApJ, 742, 76

Haarsma, D. B., Leisman, L., Donahue, M., Bruch, S., Böhringer, H., Croston, J. H., Pratt, G. W., Voit, G. M., Arnaud, M., \& Pierini, D. 2010, ApJ, 713, 1037

Iben, Jr., I. \& Renzini, A. 1983, ARA\&A, 21, 271

Johnson, R., Finoguenov, A., Ponman, T. J., Rasmussen, J., \& Sanderson, A. J. R. 2011, MNRAS, 413, 2467

Katz, N., Weinberg, D. H., \& Hernquist, L. 1996, ApJS, 105, 19

Kauffmann, G. et al. 2003, MNRAS, 341, 54

Kravtsov, A. V. 2013, ApJ, 764, L31

Kravtsov, A. V. \& Gnedin, O. Y. 2005, ApJ, 623, 650

Kriek, M. et al. 2006, ApJ, 645, 44

Lake, G. \& Schommer, R. A. 1984, ApJ, 280, 107

Leitner, S. N. \& Kravtsov, A. V. 2011, ApJ, 734, 48

Leroy, A. K., Walter, F., Brinks, E., Bigiel, F., de Blok, W. J. G., Madore, B., \& Thornley, M. D. 2008, AJ, 136, 2782

Maoz, D., Mannucci, F., \& Brandt, T. D. 2012, MNRAS, 426, 3282

Martig, M., Bournaud, F., Teyssier, R., \& Dekel, A. 2009, ApJ, 707, 250

Martin, D. C., Seibert, M., Neill, J. D., Schiminovich, D., Forster, K., Rich, R. M., Welsh, B. Y., Madore, B. F., Wheatley, J. M., Morrissey, P., \&

Barlow, T. A. 2007, Nature, 448, 780

Martizzi, D., Jimmy, Teyssier, R., \& Moore, B. 2014, MNRAS, 443, 1500

Mathews, W. G. 1990, ApJ, 354, 468

Mathews, W. G. \& Baker, J. C. 1971, ApJ, 170, 241

Matsushita, K. 2001, ApJ, 547, 693

McDonald, M., Veilleux, S., Rupke, D. S. N., Mushotzky, R., \& Reynolds, C. 2011, ApJ, 734, 95

McNamara, B. R. \& Nulsen, P. E. J. 2007, ARA\&A, 45, 117

Nagino, R. \& Matsushita, K. 2009, A\&A, 501, 157

Negri, A., Ciotti, L., \& Pellegrini, S. 2014, MNRAS, 439, 823

Onodera, M. et al. 2012, ApJ, 755, 26

Rasmussen, J. \& Ponman, T. J. 2007, MNRAS, 380, 1554

Salim, S., Fang, J. J., Rich, R. M., Faber, S. M., \& Thilker, D. A. 2012, ApJ, 755,105

Sasaki, T., Matsushita, K., \& Sato, K. 2014, ApJ, 781, 36

Stinson, G., et. al. 2006, MNRAS, 373, 1074

Sun, M., Voit, G. M., Donahue, M., Jones, C., Forman, W., \& Vikhlinin, A. 2009, ApJ, 693, 1142

Sutherland, R. S. \& Dopita, M. A. 1993, ApJS, 88, 253

van der Wel, A. et al. 2014, ApJ, 788, 28

van Dokkum, P. G., Kriek, M., \& Franx, M. 2009, Nature, 460, 717

Vikhlinin, A., Kravtsov, A., Forman, W., Jones, C., Markevitch, M., Murray, S. S., \& Van Speybroeck, L. 2006, ApJ, 640, 691

Vogelsberger, M., Genel, S., Sijacki, D., Torrey, P., Springel, V., \&

Hernquist, L. 2013, MNRAS, 436, 3031

Voit, G. M. \& Donahue, M. 2011, ApJ, 738, L24

Whitaker, K. E., Kriek, M., van Dokkum, P. G., Bezanson, R., Brammer, G., Franx, M., \& Labbé, I. 2012, ApJ, 745, 179

Whitaker, K. E. et al. 2013, ApJ, 770, L39 